\title{
Season of birth and the risk of hip fracture in Danish men and women aged $65+$
}

\section{Bo Abrahamsen ${ }^{1,2}$, Berit L. Heitmann ${ }^{3}$ and Pia A. Eiken ${ }^{4}$}

${ }^{1}$ Department of Medicine F, Gentofte Hospital, Copenhagen, Denmark

${ }^{2}$ Odense Patient data Explorative Network, Institute of Clinical Research, University of Southern Denmark, Odense, Denmark

${ }^{3}$ Research Unit for Dietary Studies, Institute of Preventive Medicine, Copenhagen University Hospital, Copenhagen, Denmark

${ }^{4}$ Department of Endocrinology and Cardiology, Hillerød Hospital, Hillerød, Denmark

\section{Edited by:}

William Duncan Fraser, University of Liverpool/Royal Liverpool University Hospital, UK

Reviewed by:

Elaine Dennison, MRC Lifecourse Epidemiology Unit, UK

Muhammad Kassim Javaid,

University of Oxford, UK

*Correspondence:

Bo Abrahamsen, Department of Medicine F, Gentofte Hospital,

Copenhagen DK-2900, Denmark. e-mail: b.abrahamsen@physician.dk
Vitamin D status in pregnant women has been linked to childhood bone mineral density in their offspring but it is unclear if effects extend to fracture risk in adulthood or even old age. As vitamin D levels in the population show pronounced seasonal variation in Denmark, we performed an epidemiological analysis of hip fracture rates as a function of season of birth, age, and sex. We retrieved information on all hip fractures in the 9-year period between 1997 and 2005 in all men and women aged 65-95, excluded hip fractures that occurred in current and recent prednisolone users, and subsequently calculated fracture rates and relative risks. The analysis covered 541,109 men and 691,522 women. In women, we observed a small but statistically significant difference between fracture rates by season of birth for all age intervals expect the youngest (age 65-69). A similar pattern was seen in men, but this was only statistically significant in the two oldest age groups (age 85-89 and 90-95). These findings suggest that vitamin D availability in the first and second trimester of intrauterine life could have a small but lasting impact on bone health and the risk of osteoporotic fractures. Further studies are needed.

Keywords: epidemiology, uterine factors, hip fracture, osteoporosis, nutrition, fractures, geriatric medicine, endocrinology

\section{BACKGROUND}

Maternal vitamin D status during pregnancy has been linked to the bone mineral density of growing children (Javaid et al., 2006). Both adult bone mass and hip fractures is linked to childhood weight and growth trajectory (Cooper et al., 2001; Javaid et al., 2011), but it remains unclear if there is a long term effect of intrauterine vitamin D status factors on the risk of fractures in adulthood or old age (Harvey and Cooper, 2004; Cooper et al., 2005; Tobias et al., 2005; Holroyd et al., 2011). In Northern Europe, serum vitamin D levels show a pronounced seasonal variation with deficiency being common in late winter and spring (Holick, 1996; Brot et al., 2001). The reason for this is that cutaneous vitamin D synthesis does not occur in winter or spring at the latitude of Denmark. Hence vitamin D sufficiency is entirely dependent on diet and on the use of vitamin D supplements. Vitamin D fortification of foods is not currently used in Denmark, though fortification has been used in time periods in the past (Osler and Heitmann, 1998). As hip fracture rates in Denmark are among the highest in the world, and disease registers are highly developed, this provides an opportunity for indirectly exploring the importance of maternal, and hence offspring fetal vitamin D status through investigating if hip fracture risk in the elderly has any relation to season of birth. Accordingly, we undertook a national register based study of hip fracture occurrence in relation to age, gender, and season of birth over a 9-year period among Danish residents aged 65-95 years. The study hypothesis was that fracture rates would be lower in persons born in the last two quarters of the year as their period of maximum femur mineralization in utero would take place in
Summer and Autumn where maternal vitamin D levels would be at their highest.

\section{STUDY POPULATION AND METHODS}

The study outcome was hospitalization with a hip fracture diagnosis (ICD-10 codes S720 or S721) in the period January 1, 1997 to December 31, 2005 as registered in the Danish Hospital Discharge Register (Landspatientregistret) in persons aged between 65 and 95 years (included), who had not filled a prescription for prednisolone within the last year. The hospital discharge register holds information about all hospital diagnoses in Danish residents and is linked to the National Prescription Register, which holds prescription information for all Danish pharmacies. Access was provided through Statistics Denmark, reference no 702538. For patients who had more than one hip fracture admission we only counted the first admission in order to avoid inflating the fracture risk estimates by including readmissions.

The number of persons alive at the beginning of each calendar year (i.e., outcome year), categorized into month and year of birth was obtained from Statistics Denmark in order to serve as the denominator for calculation of fracture rates. A total of 541,109 men and 691,522 women contributed to the analysis (i.e., were in the age interval 65-95 years for part or the whole period 19972005 ), with 334,704 men and 476,560 women forming the study population on January 1, 1997.

The study population was stratified by gender and divided into 5 -year age intervals. Adjusted fracture rates were calculated to take into account also the small age difference between subjects born 
in different quarters of the same year - e.g., subjects born later in the year are younger than subjects born earlier in the year. We used conventional descriptive statistics and a two-sided critical $p$-value of 0.05 .

The $\mathrm{H}_{\mathrm{o}}$ hypothesis was that the risk of hip fracture was independent of the quarter of the year that persons were born $(\mathrm{Q} 1$, Q2, Q3, or Q4) within each age and sex stratum. This was tested using $\chi^{2}$ tests. Additional exploratory tests were done using multiple linear regression analysis with fracture rates as the dependent variables after natural log transformation, with age, fracture year, and dummy variables representing quarter of year as independent variables. The aim of this procedure was to control for the effects rates attributable to age and to temporal trends in fracture incidence. We used SPSS versions 19.0 and 11.0.

\section{RESULTS}

The risk of hip fracture increased exponentially in both genders (Tables 1A,B), from 2 per 1000 patients years (py) in 65- to 69year-old women to 40 per 1000 py in women aged 90-95, and from $1 / 1000$ py in men aged $65-69$ to $40 / 1000$ py in men aged 90-95. This amounted to a threefold increase in fracture risk for every 10 year increase in age. There was a clear temporal trend with declining age specific rates of hip fractures in both men $(3.2 \%$ per calendar year) and women (5.1\% per calendar year) over the study period.

\section{WOMEN}

In women $69 \%$ of hip fractures occurred in persons aged $80+$. We observed a small but statistically significant difference between fracture rates by season of birth for all age intervals expect the youngest, i.e., age 65-69 (Table 1A; Figure 1). Contrary to our expectations, persons born in the first quarter of the year had the lowest risk of hip fracture. This was confirmed by multiple regression analysis, showing a $4 \%$ higher rate in women born in Q2 $(p<0.05)$ and Q3 $(p<0.05)$, compared with those born in the first quarter of the year (Table 2).

\section{MEN}

Seventy percent of hip fractured occurred in those aged $80+$. Fracture rates differed weakly but significantly by season of birth only for the two oldest age groups, age 85-89 and 90-95, while we found no significant difference in the younger age groups. The highest fracture rate was seen in men born in Q4, this was $8 \%$ higher than in Q1 $(p<0.05)$. For Q2 and Q3, rates were similar and about 3\% higher than in Q1. However, this difference was not statistically significant $(p=0.18-0.26)$.

\section{DISCUSSION}

This study shows an association between season of birth and the risk of hip fracture in elderly women and men in Denmark, a geographical region with strong variation in serum vitamin $\mathrm{D}$ levels through the year. Cutaneous vitamin $\mathrm{D}$ synthesis does not occur during 6-7 months of the year due to too low UVB levels at the latitude of Denmark (54-58 ${ }^{\circ}$; Holick, 1996; Brot et al., 2001). The effect was small - amounting to $4-8 \%$ in relative risk - and thus considerably weaker than, e.g., the influence of age, BMD, or a family history of fracture. On the other hand, unlike these factors, vitamin D levels are easily modifiable.

Table 1 | Hip fractures in women.

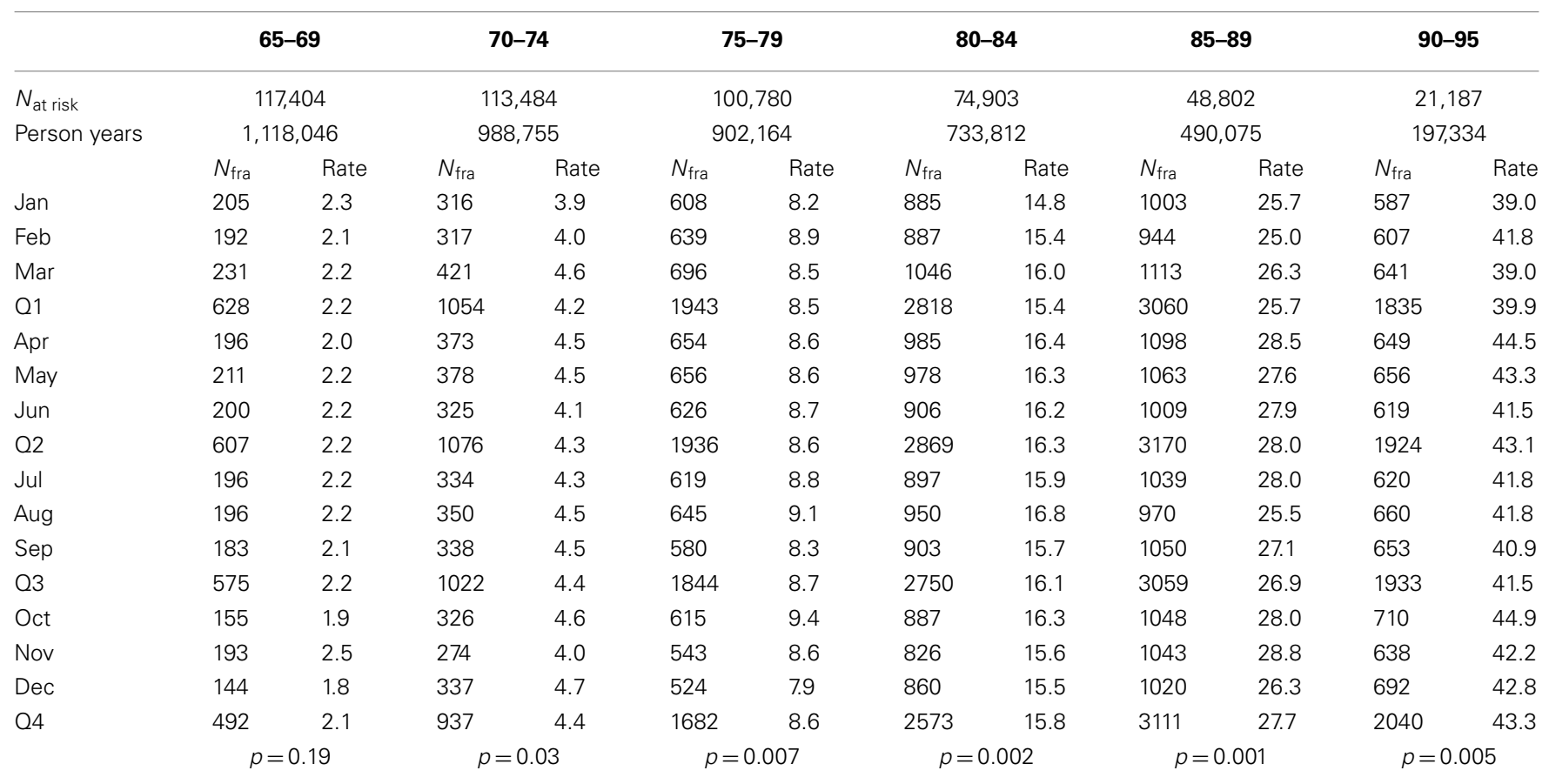

Women. Hip fractures in Danish women 1997-2005 as a function of age and season of birth. Fracture rates are in fractures per 1000 person years, adjusted for the

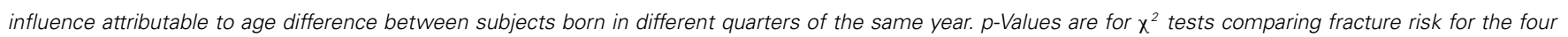
quarters of the year. Number of subjects per age group is per January 1, 1997 as size of population at risk varies by outcome year. 


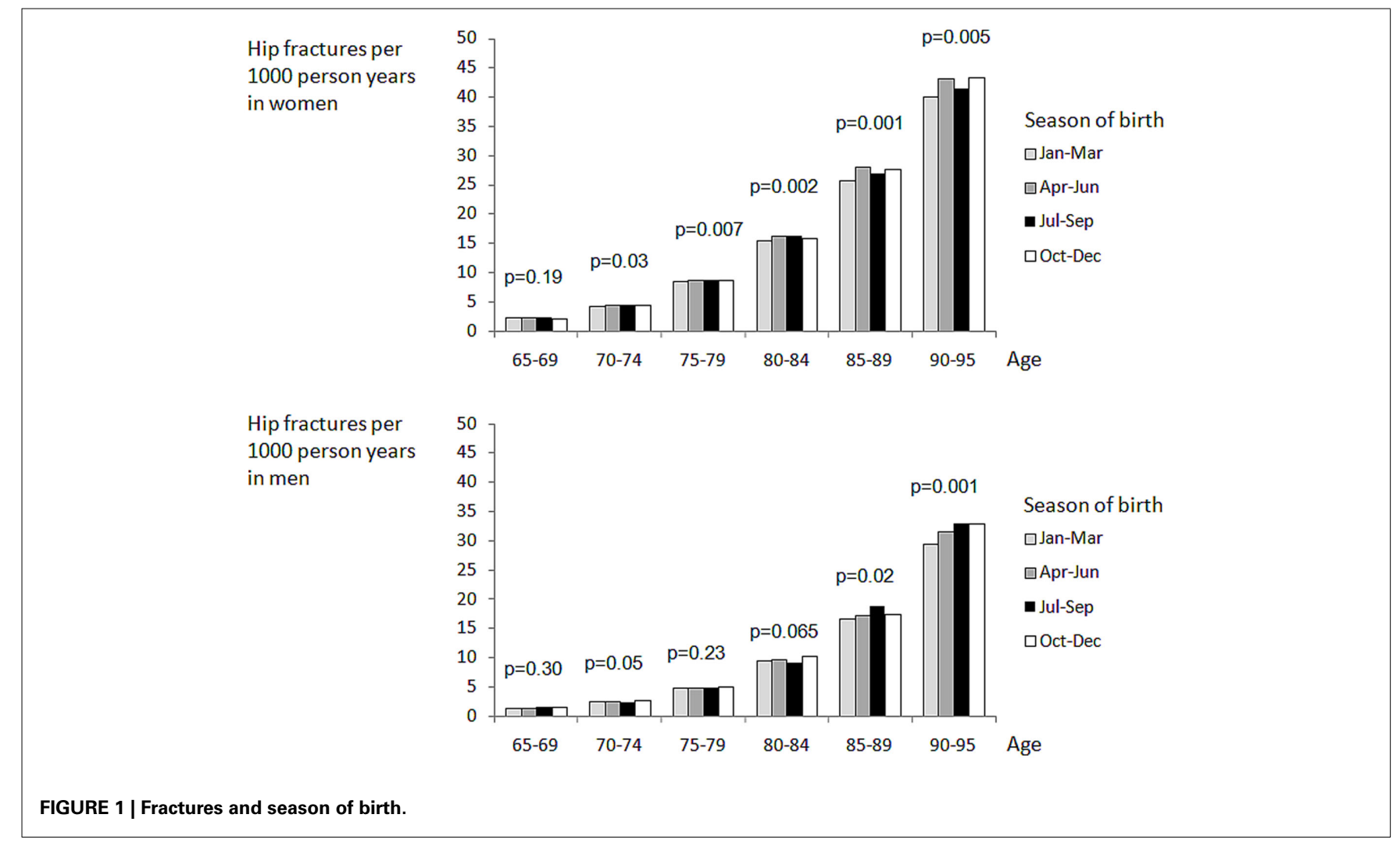

Table 2 | Hip fractures in men.

\begin{tabular}{|c|c|c|c|c|c|c|c|c|c|c|c|c|}
\hline & \multicolumn{2}{|c|}{$65-69$} & \multicolumn{2}{|c|}{$70-74$} & \multicolumn{2}{|c|}{$75-79$} & \multicolumn{2}{|c|}{$80-84$} & \multicolumn{2}{|c|}{$85-89$} & \multicolumn{2}{|c|}{$90-95$} \\
\hline$N_{\text {at risk }}$ & \multicolumn{2}{|c|}{104,168} & \multicolumn{2}{|c|}{90,068} & \multicolumn{2}{|c|}{69,476} & \multicolumn{2}{|c|}{42,820} & \multicolumn{2}{|c|}{21,388} & \multicolumn{2}{|c|}{6,785} \\
\hline Person years & \multicolumn{2}{|c|}{787,422} & \multicolumn{2}{|c|}{638,587} & \multicolumn{2}{|c|}{507,435} & \multicolumn{2}{|c|}{337,369} & \multicolumn{2}{|c|}{175,148} & \multicolumn{2}{|c|}{144,333} \\
\hline Jan & 94 & 1.1 & 162 & 2.4 & 236 & 4.3 & 311 & 8.6 & 262 & 13.9 & 587 & 27.6 \\
\hline Feb & 126 & 1.5 & 190 & 2.8 & 284 & 5.4 & 364 & 10.5 & 361 & 20.0 & 607 & 30.9 \\
\hline Apr & 116 & 1.3 & 166 & 2.4 & 292 & 5.2 & 357 & 9.9 & 303 & 16.6 & 649 & 33.0 \\
\hline May & 110 & 1.2 & 192 & 2.7 & 259 & 4.6 & 330 & 9.1 & 277 & 15.2 & 656 & 32.4 \\
\hline Jun & 128 & 1.6 & 171 & 2.6 & 249 & 4.7 & 334 & 10.1 & 344 & 19.8 & 619 & 29.1 \\
\hline Q2 & 354 & 1.4 & 529 & 2.5 & 800 & 4.9 & 1021 & 9.7 & 924 & 17.2 & 1924 & 31.5 \\
\hline Jul & 110 & 1.4 & 156 & 2.4 & 240 & 4.6 & 306 & 9.0 & 351 & 19.9 & 620 & 36.8 \\
\hline Nov & 98 & 1.4 & 169 & 3.0 & 240 & 5.2 & 330 & 10.5 & 301 & 17.9 & 638 & 36.4 \\
\hline Dec & 99 & 1.3 & 152 & 2.5 & 250 & 5.2 & 320 & 9.7 & 329 & 17.8 & 692 & 34.1 \\
\hline \multirow[t]{2}{*}{$\mathrm{Q} 4$} & 316 & 1.4 & 476 & 2.7 & 701 & 4.9 & 990 & 10.2 & 921 & 17.3 & 2040 & 32.8 \\
\hline & \multicolumn{2}{|c|}{$p=0.30$} & \multicolumn{2}{|c|}{$p=0.05$} & \multicolumn{2}{|c|}{$p=0.23$} & \multicolumn{2}{|c|}{$p=0.065$} & \multicolumn{2}{|c|}{$p=0.02$} & \multicolumn{2}{|c|}{$p=0.001$} \\
\hline
\end{tabular}

Men. Hip fractures in Danish men 1997-2005 as a function of age and season of birth. Fracture rates are in fractures per 1000 person years, adjusted for the influence attributable to age difference between subjects born in different quarters of the same year. $p$-Values are for $\chi^{2}$ tests comparing fracture risk for the four quarters of the year. Number of subjects per age group is per January 1, 1997 as size of population at risk varies by outcome year. 
The risk was highest in persons born in Q2 and Q3, pointing toward greater importance of maternal vitamin $\mathrm{D}$ levels in the second than third trimester, despite the high rate of femur mineralization that has been shown to take place toward the end of pregnancy and the relationship between maternal vitamin $\mathrm{D}$ levels in late pregnancy and childhood BMD discussed below (Table 3).

While these findings suggest that maternal vitamin D status in the first and second trimester of pregnancy could influence the risk of fractures in late adulthood, more research is needed. It may be possible to further investigate this in other study populations and countries and perhaps also explore information on fortification programs and historical data on sunshine hours to confirm or refute the hypothesis that the current findings lead to.

Existence of a link between maternal vitamin D status and childhood bone mineral density was convincingly demonstrated by Javaid et al. (2006), by measuring BMD by DXA in 198 9-yearold children, whose mothers had their serum $25 \mathrm{OH}$-vitamin D levels measured in the last weeks of the third trimester of pregnancy. A lower BMD at the spine and total body was found for children born to women with low vitamin $\mathrm{D}$ status and a direct relationship was further supported by demonstrating a significant correlation between umbilical blood calcium and subsequent BMD. This does not rule out a key role of vitamin D levels at an earlier time in pregnancy as low vitamin D level in late pregnancy is likely to be associated also with an increased likelihood of low vitamin D levels in the first and second trimester. Indeed, maternal vitamin D status determined at 34 weeks of gestation was significantly associated with femoral metaphyseal cross-sectional area and a femoral splaying index measured by ultrasound, not only at the same measurement time but also with these ultrasound measurements done earlier, at 19 weeks of pregnancy. This indirectly supports the assumption that women who were vitamin D deficient at 34 weeks must also have been low in vitamin D earlier in pregnancy, to explain skeletal differences in the fetus as early as at 19 weeks.

As the youngest subjects in our analysis were born in 1940 and the oldest in 1902, there is room for pronounced cohort and period effects. A non-exhaustive list of potentially contributing factors includes two world wars, periods of national dietary fortification with calcium and/or vitamin D (Osler and Heitmann, 1998), introduction of hormone replacement therapy and changes in alcohol consumption, smoking, exercise, and obesity. These factors may contribute to the long term temporal trends in fracture epidemiology but are unlikely to exert seasonal influences on vitamin D status. Important sources of vitamin D are fish, meat, and vitamin supplements. Though these factors may show have seasonal variation, the variation appears too small to offset the influence of sunlight. Thus, meat and fish eaters have higher average serum vitamin $D$ levels than vegetarians and vegans, but serum vitamin $\mathrm{D}$ levels show the same variation over the year (Crowe et al., 2011). As previously shown and discussed more comprehensively (Abrahamsen and Vestergaard, 2010), age adjusted hip fracture rates have been declining in Denmark in the past decade as in many other Western countries (Jaglal et al., 2005; Kannus et al., 2006; Chevalley et al., 2007; Abrahamsen and Vestergaard, 2010).
The present study has important strengths in that we were able to remove from the analysis fractures that occurred followed prednisolone use, an important environmental modifier of fracture risk. Also, we used conservative fracture rates because we allowed only one hip fracture - the first one to occur within the study period - per patient to enter the analysis. This reduces the likelihood of the strength of the association being overestimated due to recurrent fractures or fracture complications.

There are also limitations to this epidemiological study that should be borne in mind. We had access to detailed information only on fracture patients, not on the background population. Because of this, we could not conduct a register based cohort study and analyze the time to fracture information using Cox regression analysis, taking into account dates of fracture, dates of death, and date of end of observations. Thus, we relied on population census information for the first of January in each outcome year, broken down by year and month of birth. We assumed that deaths were evenly distributed over the year and that season of birth did not exert an influence on survival beyond the effect on age itself. The effects found were very small and we cannot rule out the possibility that they could be the affected by subtle fluctuations in at risk population death rates uncaptured by our method. Also, we did not have information about loss of follow-up due to emigration, but assume that the effect would be small in elderly study populations. Fractures sustained and treated on foreign holidays would also not be captured.

Results in men and women differed slightly, with birth in Q4 being associated with increased fracture risk for men but not for women. However, Q1 was associated with low risk in both genders and Q2 and Q3 with slightly raised risk. This is unlikely to be a true sexual dimorphism in the role of intrauterine vitamin D but probably reflects random variation due to the lower number of hip fractures in men and smaller number of $80+$ year olds.

We deliberately did not adjust results for prior fractures, though prior fractures are strong risk factors for hip fracture. First, we aimed to explore a biological hypothesis, not develop a risk factor

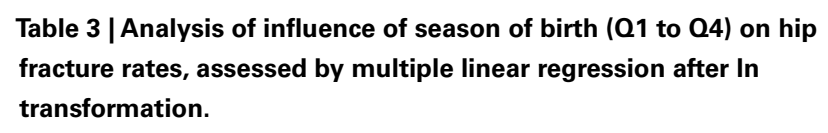

\begin{tabular}{lll}
\hline & $\operatorname{Exp}(\mathbf{B})$ & $\boldsymbol{p}$ \\
\hline WOMEN & & \\
Q1 & 1 & Reference \\
Q2 & $1.04(1.00-1.07)$ & 0.04 \\
Q3 & $1.04(1.00-1.08)$ & 0.04 \\
Q4 & $1.02(0.98-1.06)$ & 0.29 \\
MEN & & \\
Q1 & 1 & Reference \\
Q2 & $1.03(0.99-1.09)$ & 0.18 \\
Q3 & $1.03(0.98-1.08)$ & 0.26 \\
Q4 & $1.08(1.03-1.13)$ & 0.003 \\
\hline
\end{tabular}

Three dummy variables were formed to capture differences in rates between quarters of birth year with $Q 1$ as reference. Results were mutually adjusted and controlled for age and outcome year. 
Table 4 | Relationship between season of birth and the season coinciding with the three trimesters (A) and with the period of exponential mineralization of the femur in utero (B).

\begin{tabular}{|c|c|c|c|}
\hline Season of birth & Conception & $\begin{array}{l}\text { (A) Trimesters coinciding with } \\
\text { summer and autumn }\end{array}$ & $\begin{array}{l}\text { (B) Period of exponential femur } \\
\text { mineralization in utero (week 20-36) }\end{array}$ \\
\hline Jan-Mar (Q1) & Apr-Jun & 2nd, partly 3rd & Sep-Mar (winter) \\
\hline Apr-Jun (Q2) & Jul-Sep & Partly 2nd & Dec-Jun (spring) \\
\hline Jul-Sep (Q3) & Oct-Dec & $3 r d$ & Mar-Sep (summer) \\
\hline Oct-Dec (O4) & Jan-Mar & 3rd, partly 2nd & Jun-Dec (autumn) \\
\hline
\end{tabular}

The highest serum vitamin D levels in Danish women are found from July to October (Brot et al., 2001). The lowest average second trimester vitamin D levels in utero would be experienced by persons born in $Q 3$ and most of Q2, and the lowest average third trimester levels by persons born in Q2 and most of Q1. In a person born in April (Q2), the period of maximum mineralization of the femur would begin in early December and proceed through spring where vitamin D levels in the population are at their lowest. By contrast, persons born in December (Q4) enter their period of exponential mineralization in August where vitamin D levels are at their highest.

algorithm. Adjusting results for early manifestations of disease in the form of non-hip fractures could bias findings, similar to attempting to correct for chronic bronchitis when exploring a relationship between smoking and pulmonary cancer. Second, hip fractures are the most reliably adjudicated fracture type in our health registers (Hundrup et al., 2004), chiefly because this type of fracture always leads to hospitalization and usually to surgery.

In summary, hip fracture risk was found to be a few percent higher in women born in the second and third quarter of the

\section{REFERENCES}

Abrahamsen, B., and Vestergaard, P. (2010). Declining incidence of hip fractures and the extent of use of anti-osteoporotic therapy in Denmark 1997-2006. Osteoporos. Int. 21, 373-380.

Brot, C., Vestergaard, P., Kolthoff, N., Gram, J., Hermann, A. P., and Sorensen, O. H. (2001). Vitamin D status and its adequacy in healthy Danish perimenopausal women: relationships to dietary intake, sun exposure and serum parathyroid hormone. Br. J. Nutr. 86(Suppl. 1), S97-S103.

Chevalley, T., Guilley, E., Herrmann, F. R., Hoffmeyer, P., Rapin, C. H., and Rizzoli, R. (2007). Incidence of hip fracture over a 10 -year period (1991-2000): reversal of a secular trend. Bone 40, 1284-1289.

Cooper, C., Eriksson, J. G., Forsen, T., Osmond, C., Tuomilehto, J., and Barker, D. J. (2001). Maternal height, childhood growth and risk of hip fracture in later life: a longitudinal study. Osteoporos. Int. 12, 623-629.

Cooper, C., Javaid, K., Westlake, S., Harvey, N., and Dennison, E. (2005). Developmental origins of osteoporotic fracture: the role of maternal vitamin D insufficiency. J. Nutr. 135, 2728S-2734S

Crowe, F. L., Steur, M., Allen, N. E., Appleby, P. N., Travis, R. C., and
Key, T. J. (2011). Plasma concentrations of 25-hydroxyvitamin $\mathrm{D}$ in meat eaters, fish eaters, vegetarians and vegans: results from the EPICOxford study. Public Health Nutr. 14, 340-346.

Harvey, N., and Cooper, C. (2004). The developmental origins of osteoporotic fracture. J. Br. Menopause Soc. 10, 14-15.

Holick, M. F. (1996). Vitamin D and bone health. J. Nutr. 126(Suppl. 4), 1159S-1164S.

Holroyd, C., Harvey, N., Dennison, E., and Cooper, C. (2011). Epigenetic influences in the developmental origins of osteoporosis. Osteoporos. Int. PMID: 21656266. [Epub ahead of print].

Hundrup, Y. A., Hoidrup, S., Obel, E. B., and Rasmussen, N. K. (2004). The validity of self-reported fractures among Danish female nurses: comparison with fractures registered in the Danish National Hospital Register. Scand. J. Public Health 32, 136-143.

Jaglal, S. B., Weller, I., Mamdani, M., Hawker, G., Kreder, H., Jaakkimainen, L., and Adachi, J. D. (2005). Population trends in BMD testing, treatment, and hip and wrist fracture rates: are the hip fracture projections wrong? J. Bone Miner. Res. 20, 898-905.

year, suggesting that vitamin $\mathrm{D}$ availability in the first and second trimester of intrauterine life may have a lasting impact on bone health and the risk of osteoporotic fractures. The findings were less pronounced in men, but for both genders the risk of hip fracture was at its lowest in subjects born in the first quarter of the year, coinciding with good maternal vitamin D status in the second trimester. Though interesting, these findings should first be confirmed in other large datasets before a conclusion is made.

Javaid, M. K., Crozier, S. R., Harvey, N. C., Gale, C. R., Dennison, E. M., Boucher, B. J., Arden N. K., Godfrey, K. M., Cooper, C., and Princess Anne Hospital Study Group. (2006). Maternal vitamin D status during pregnancy and childhood bone mass at age 9 years: a longitudinal study. Lancet 367, 36-43.

Javaid, M. K., Eriksson, J. G., Kajantie, E., Forsen, T., Osmond, C., Barker, D. J., and Cooper, C. (2011). Growth in childhood predicts hip fracture risk in later life. Osteoporos. Int. 22, 69-73.

Kannus, P., Niemi, S., Parkkari, J., Palvanen, M., Vuori, I., and Jarvinen, M. (2006). Nationwide decline in incidence of hip fracture. J. Bone Miner. Res. 21, 1836-1838.

Osler, M., and Heitmann, B. L. (1998). Food patterns, flour fortification, and intakes of calcium and vitamin D: a longitudinal study of Danish adults. J. Epidemiol. Community Health 52, 161-165.

Tobias, J. H., Steer, C. D., Emmett, P. M., Tonkin, R. J., Cooper, C., and Ness, A. R. (2005). Bone mass in childhood is related to maternal diet in pregnancy. Osteoporos. Int. 16, 1731-1741.

Conflict of Interest Statement: The study received grant support from
Kaptajnløjtnant Harald Jensen og Hustrus Fond, Denmark. The funding source had no influence on the study and did not review the manuscript. Disclosures for individual authors are as follows: Bo Abrahamsen: Grant/Research support from Novartis, Nycomed, Amgen, Merck. Speakers Bureau with Nycomed, Merck, Eli Lilly. Pia A. Eiken: Grant/Research support from Nycomed, Amgen, Novartis Speakers Bureau with Nycomed, Novartis, GSK, and Eli Lilly.

Received: 13 September 2011; paper pending published: 16 October 2011; accepted: 04 January 2012; published online: 18 January 2012.

Citation: Abrahamsen B, Heitmann BL and Eiken PA (2012) Season of birth and the risk of hip fracture in Danish men and women aged 65+. Front. Endocrin. 3:2. doi: 10.3389/fendo.2012.00002

This article was submitted to Frontiers in Bone Research, a specialty of Frontiers in Endocrinology.

Copyright (c) 2012 Abrahamsen, Heitmann and Eiken. This is an open-access article distributed under the terms of the Creative Commons Attribution Non Commercial License, which permits noncommercial use, distribution, and reproduction in other forums, provided the original authors and source are credited. 